\title{
Application of Pigeon Inspired Optimization for Multi-dimensional Knapsack Problem
}

\author{
F. Setiawan*, A. Sadiyoko, and C. Setiardjo
}

\begin{abstract}
The multi-dimensional knapsack problem (MKP) is a generalization of the classical knapsack problem, a problem for allocating a resource/subset of objects that maximize profit while not violating the capacity of the knapsack. The MKP has many practical applications in different areas and is classified as an NP-hard problem. An analytic method like a branch and bound and dynamic programming can yield the optimum solution, but its computational time growth is exponentially proportional to the problem's size. Some heuristics/metaheuristics procedure has been developed to obtain a near-optimal solution within reasonable computational times. In this paper, a pigeon-inspired optimization (PIO) is proposed for solving MKP. PIO is one of the metaheuristic algorithms that is classified in population-based swarm intelligence that is developed based on the behavior of the pigeon to find its home. However, it had gone far away from its home. In this paper, PIO implementation to solve MKP is applied to two different characteristic cases in a total of 10 cases. The result of implementing the two-best combination of parameter values for 10 cases compared to particle swarm optimization, intelligent water drop algorithm, and the genetic algorithm give satisfactory results.
\end{abstract}

Keywords: metaheuristics, multi-dimensional knapsack problem, pigeon inspired optimization, swarm intelligence.

Article Info: Received November 20, 2020; Revised March 17, 2021; Accepted June 7, 2021.

\section{INTRODUCTION}

The multi-dimensional knapsack problem (MKP) is a generalization of the knapsack problem, a problem for allocating a resource/subset of objects that maximize profit while not violating the capacity of knapsack (Martello and Toth, 1990). It explained the classical knapsack problem as suppose a hitch-hiker has to fill up his knapsack by selecting from among various possible objects that will give him maximum comfort. Another example, the knapsack problem will provide the optimal solution related to investment. Suppose that someone is going to invest a number of dollars and consider some possible alternative investments. Using this method, he can calculate profit gained from investment and how much money is required to invest.

Recently, knapsack problems are an interesting topic in research. Examples of industrial knapsack problems are capital budgeting, cargo loading, and cutting stock
(Martello and Toth, 1990). Since MKP is a generalization of the classical knapsack problem, it is more practical, and it can model all of the problems that can be modeled by the knapsack problem. According to Haddar et al. (2016), MKP has been used as a model for many real applications such as cutting stock problems, project selection, cargo loading problems, capital budgeting, allocating processors and databases in a distributed computer system, and daily management of a satellite.

Since MKP is an NP-hard problem, numerous methods have been developed by the researchers that are classified into exact and approximation methods. Exact method, such as branch and bound algorithm (Shih, 1979; Vimont et al., 2008; Manzini and Speranza, 2012); dynamic programming (Gilmore and Gomory, 1966; Weingartner and Ness, 1967); hybrid dynamic programming methods (Bertsimas and Demir, 2002; Balev et al., 2008) could generate the optimal solution,

\footnotetext{
F. Setiawan* is with the Department of Industrial Engineering, Universitas Katolik Parahyangan, Indonesia (email: fransetiawan@unpar.ac.id).

* Corresponding author
} 
but they can solve only instances of very limited size in an acceptable computation time. Heuristics/metaheuristics procedure could obtain a nearoptimal solution within reasonable computational times compared to the analytic method. There are two classifications of approximation methods, heuristics, and metaheuristics. Heuristics and metaheuristics have recently become a focus of researchers for solving MKP (Haddar et al., 2016). The heuristic approach has been developed by Battiti and Vecchiolli (1994) and Freville and Plateau (1986). Heuristics are problem-dependent that are designed and applicable to a problem. The research on the application of metaheuristic method to solve MKP are tabu search (Vasquez and Hao, 2001; Dammeyer and Voss, 1993, Glover and Kochenberger, 1996; Vasquest and Vimont, 2005, Lai et al., 2018); genetic algorithm (Khuri et al., 1994; Chu and Beasley, 1998; Berberler et al., 2013; Martins et al., 2014); simulated annealing (Leung et al., 2012; Rezoug et al., 2015); ant colony optimization (Kong et al., 2008; Ke et al., 2010; Fingler et al., 2014); particle swarm optimization (Kong et al., 2006; Hembecker et al., 2007; Wan and Nolle, 2009; Chen et al., 2010; Ktari and Chabchoub, 2013); Tisna, 2013, Chih, 2015, Haddar et al., 2016); intelligent water drops (Shah-Hosseini, 2009), binary artificial algae algorithm (Zhang et al., 2016), binary multi-verse optimizer (Baseet et al., 2019), modified flower pollination (Basset et al., 2018).

This paper will propound a new evolutionary computation technique, Pigeon Inspired Optimization (PIO) (Duan and Qiao, 2014), to answer MKP. PIO is a novel bio-inspired computation algorithm, which was inspired by the homing characteristics of pigeons (Duan and $\mathrm{Li}, 2014)$. PIO has been used to solve continuous air robot path planning problems (Duan and Qiao, 2014), target detection task for Unmanned Aerial Vehicles (UAVs) at low attitude (Duan and Li, 2014), control parameter design for automatic carrier landing system (Duan and Deng, 2016), prediction control for unmanned air vehicles (Dou and Duan, 2016).

Studies about the application of PIO for solving MKP have been done by Bolaji et al., 2017 and Bolaji et al., 2020. Bolaji et al. (2017) proposed a binary PIO for solving MKP. They used n-bit binary string representation to represent the MKP solution and used the penalty function method to tackle the MKP's constraints. Bolaji et al. (2020) developed a modified binary PIO for solving MKP, which integrated a crossover procedure of evolutionary algorithm in order to improve the solution. The crossover procedure is embedded in the landmark operator in the PIO. The result was that the modified binary PIO was outperformed the binary PIO.

This research is the same as what is proposed by Bolaji et al. (2017). In this paper, we propose another way to represent the MKP solution and to handling the MKP's constraints. We do not use the special 'binary' term of the PIO; however, we use the random value and use the sigmoid function to convert the random value to 0-1 (binary). We use the drop/add item procedure and local heuristic method, namely Heuristic Undesirability (HUD), to handle the MKP's constraints instead of the penalty function. Another difference is that we use ten instances from Senyu and Toyada (1967) and Weingartner and Ness (1967) and compare the PIO performance from particle swarm optimization (PSO), intelligent water drops (IWD), and genetic algorithm (GA).

\section{LITERATURE REVIEW}

In this section, we describe multi-dimensional knapsack problem (MKP), pigeon-inspired optimization, and methods used in this research.

\subsection{Multi-dimensional knapsack problem}

The multi-dimensional knapsack problem (MKP) is a problem for allocating a resource/subset of objects to maximize profit without violating the capacity of knapsack (Martello and Toth, 1990). There are a set of $n$ items which each item has $m$ weight. Every item has a profit/a benefit $p_{i}(i=1, \ldots, n)$, various weight $W_{j i}$ for every single knapsack $j$, and there is a knapsack of $m$ dimensions with a capacity of each $c_{1}, \ldots, c_{m}$. The objective function is to choose a subset of items that assures the capacity of the knapsack is not exceed and yields maximum profit. The decision variable $x_{i}$ represents whether the item $i$ will be selected or not. It will value 1 if item $i$ is selected and 0 if item $i$ is not selected. MKP can be formulated as follow:

Maximize $\sum_{i=1}^{n} p_{i} \times x_{i}$

Subject to

$$
\begin{aligned}
& \sum_{j=1}^{n} w_{j i} \times x_{i} \leq c_{j}, \quad j=1, \ldots, m \\
& x_{i} \in(0,1), \quad i=1, \ldots, n
\end{aligned}
$$

\subsection{Pigeon inspired optimization}

Pigeon Inspired Optimization (PIO) is a populationbased swarm intelligence metaheuristic that is inspired by pigeon's behavior. Inspired by these, in the PIO, the map and compass operator model is based on magnetic field and sun, whereas the landmark operator model is based upon landmarks (Duan and Qiao, 2014).

\subsubsection{Map and compass operator}

In map and compass operator, every $t^{\text {th }}$ iteration will update positions, $X_{i}$, and velocities, $V_{i}$, of pigeon $i$ in a $D$ dimension search space (Duan and Qiao, 2014). The equations:

$$
\begin{aligned}
& V_{i}(t)=V_{i}(t-1) e^{-R t}+\operatorname{rand}\left(X_{g}-X_{i}(t-1)\right) \\
& \begin{array}{ll}
X_{i}(t)= & X_{i}(t-1)+V_{i}(t) \\
R \quad=\text { the map and compass factor. } \\
\text { rand } \quad=\text { random numbers from } 0 \text { to } 1 . \\
X_{g} \quad=\text { the most recent global best position } \\
\text { compared with other positions among all pigeons. }
\end{array}
\end{aligned}
$$

\subsubsection{Landmark operator}

According to Duan and Qiao (2014), in landmark 
operator, $50 \% N_{p}$ diminishes the number of pigeons for every iteration. Nevertheless, the pigeons are not close enough to the destination and not familiar with the landmarks. $X_{c}(t)$ is a midpoint pigeon's position at $t^{\text {th }}$ iteration. These equations show their update position for every iteration:

$$
\begin{aligned}
& N_{p}(t)=\frac{N_{p}(t-1)}{2} \\
& X_{c}(t)=\frac{\sum X_{i}(t) \cdot \text { fitness }\left(X_{i}(t)\right)}{N_{p} \sum \text { fitness }\left(X_{i}(t)\right)} \\
& X_{i}(t)=X_{i}(t-1)+\text { rand. }\left(X_{c}(t)-X_{i}(t-1)\right)
\end{aligned}
$$

where $N_{p}$ is the number of pigeons that is diminished at every generation, $t$ is the $t^{\text {th }}$ iteration, and $X_{c}$ is the center of some pigeon's position at the $t^{\text {th }}$ iteration, $X_{i}$ is the position of $i^{\text {th }}$ pigeon.

The fitness value in equation 7 can be altered based on the problem that will be solved. If the objective function is maximizing, then equation 9 will be used to calculate the fitness value; otherwise, equation 10 will be used. Equation 9 and 10 are given below:

$$
\begin{aligned}
\text { fitness }\left(X_{i}(t)\right) & =\frac{1}{f \min \left(\left(X_{i}(t)\right)+\epsilon^{*}\right)} \\
\text { fitness }\left(X_{i}(t)\right) & =f \max \left(X_{i}(t)\right)
\end{aligned}
$$

The details of PIO can be referred to by Duan and Qiao (2014).

\section{METHODOLOGY}

\subsection{Encoding and decoding}

Encoding and decoding is a process that is needed in applying PIO algorithm to solve MKP. Encoding is a process to translate the MKP problem to the PIO, and decoding is a process to translate the PIO inner modification process to the MKP problem solution. This $\mathrm{PIO}$ algorithm analogizes the position of pigeons in each dimension as a representation of the solution so that each pigeon is represented as a potential solution. The dimension of the pigeon in this study is items. The position of the pigeon will then be converted into binary numbers to determine whether that item is taken into the knapsack.

To find out more clearly how the encoding and decoding process in the application of PIO for MKP is explained through a simple example problem. Suppose company $\mathrm{X}$ will choose a project that will be done by company $\mathrm{X}$ in the next three years; each project has its own annual cost (the annual cost is analogous to the weight of each item). Each project also has its own profit that is obtained by the investment (the profit is analogous to the benefit of each item). Every year there is a maximum budget that is budgeted by company $\mathrm{X}$ to run the chosen project. The maximum budget is analogous to the capacity of knapsack (year 1 budget is capacity of knapsack 1, year 2 budget is capacity of knapsack 2, etc.). The maximum budget every year respectively is $\$ 25.000, \$ 15.000$ and $\$ 20.000$. The budget and profit for each project per year can be seen in Table 1.

The encoding process is done by determining the position of the pigeon in each dimension in the $0^{\text {th }}$ iteration. In this $0^{\text {th }}$ iteration, the position will be obtained from a random value between 0-1 since there is no previous position (for the next iteration, it is adjusted with the position calculation formula in PIO). Table 2 shows the result of the random value that has been obtained for each dimension.

After the random value is obtained, then the decoding process is carried out. The decoding process is done by changing the random value into a binary number, where if it has a value of 1 then the decision of goods is taken and vice versa. Changing this binary number is done through the calculation of the sigmoid function. The sigmoid function is one method of converting continuous values into binary numbers in the application of metaheuristics (Banati and Bajaj, 2011 and Palit et al., 2011). The sigmoid function calculation formula is as follows:

$$
X_{i j}=\left\{\begin{array}{l}
1, \text { if } \operatorname{rand}() \leq \frac{1}{1+\exp \left(-X_{i j}\right)} \\
0, \text { otherwise }
\end{array}\right.
$$

Table 3 below shows the binary results using the sigmoid function equation for each pigeon position or the decision of which item is taken in each dimension.

\subsection{Drop/add item}

The decoding process then continues with the drop/add item procedure. This procedure is used to handle the constraints in MKP. The way to handle these

Table 1: Budget for each project per year

\begin{tabular}{|c|c|c|c|c|}
\hline Project & $\begin{array}{c}\text { Cost in year 1 } \\
\text { (thousand \$) }\end{array}$ & $\begin{array}{c}\text { Cost in year 2 } \\
\text { (thousand \$) }\end{array}$ & $\begin{array}{c}\text { Cost in year 3 } \\
\text { (thousand \$) }\end{array}$ & $\begin{array}{c}\text { Profit } \\
\text { (thousand \$) }\end{array}$ \\
\hline A & 12 & 10 & 10 & 2000 \\
\hline B & 8 & 5 & 5 & 1200 \\
\hline C & 7 & 3 & 5 & 1000 \\
\hline D & 10 & 7 & 10 & 1500 \\
\hline
\end{tabular}

Table 2: Random value of the position of the pigeon in the $0^{\text {th }}$ position

\begin{tabular}{|c|c|c|c|c|}
\hline Dimension & D1 & D2 & D3 & D4 \\
\hline Position & 0,543 & 0,672 & 0,347 & 0,958 \\
\hline
\end{tabular}


Table 3: Decoding using the sigmoid function

\begin{tabular}{|c|c|c|c|c|}
\hline Dimension & D1 & D2 & D3 & D4 \\
\hline Position & 0,543 & 0,672 & 0,347 & 0,958 \\
\hline Binary & 1 & 1 & 0 & 1 \\
\hline
\end{tabular}

Table 4: HUD value of each project

\begin{tabular}{|c|c|c|c|c|}
\hline Dimension & D1 & D2 & D3 & D4 \\
\hline Position & 0,543 & 0,672 & 0,347 & 0,958 \\
\hline Binary & 1 & 1 & 0 & 1 \\
\hline HUD & 0.0053 & 0.0050 & 0.0050 & 0.0060 \\
\hline
\end{tabular}

Table 5: Problem characteristics for 10 cases

\begin{tabular}{|c|c|c|c|}
\hline No & Instances & Number of Knapsack & Number of Item \\
\hline 1 & SENTO1 & 30 & 60 \\
\hline 2 & SENTO2 & 30 & 60 \\
\hline 3 & WEING1 & 2 & 28 \\
\hline 4 & WEING2 & 2 & 28 \\
\hline 5 & WEING3 & 2 & 28 \\
\hline 6 & WEING4 & 2 & 28 \\
\hline 7 & WEING5 & 2 & 28 \\
\hline 8 & WEING6 & 2 & 28 \\
\hline 9 & WEING7 & 2 & 105 \\
\hline 10 & WEING8 & 2 & 105 \\
\hline
\end{tabular}

constraints in this paper is by dropping (drop procedure) or adding (add procedure) the items. If there is excess capacity, the procedure that will be done is dropping the item. Otherwise, if there is remaining capacity, the procedure that will be done is adding the items as much as possible. The local heuristic method is used for handling this constraint. The local heuristic method used is Heuristic Undesirability (HUD). Based on ShahHosseini (2008), HUD is one of the simple local heuristic methods that show how big the item is undesirable in a solution. The formula for calculating the HUD for each item is as follow:

$$
H U D(j)=\frac{1}{m b_{j}} \sum_{k=1}^{m} r_{j k}
$$

with $b_{j}$ is the profit of item $j, r_{j k}$ is the weight from item $j$ at knapsack $k$, and $m$ is the number of knapsacks. Based on equation 12 above, it is obtained the HUD value for each project/item. The procedure to handling the constraint in MKP can be done based on this value. The drop/add item procedure can be seen in the following flowchart in figure 1. Based on the flowchart, from the simple example about the choice of project in company $\mathrm{X}$, the total weight of all items chosen exceeds the capacity/maximum budget. All three projects/items chosen (projects 1,2 and 4) will be dropped one by one until the total weight of projects/items does not exceed the maximum budget/capacity from the item with the biggest HUD value. Table 4 shows the value of HUD for each project/item that is chosen for the drop item.

From table 4, it is known that the first project/item dropped is item 4 (has the biggest HUD value). After item 4 is dropped, it will be recalculated whether the project/item chosen exceeds the capacity/maximum budget. Capacity has been fulfilled when the project/item 4 is dropped, so the drop procedure process stops there. The procedure of drop/add item procedure can be seen in Figure 1.

\subsection{Pigeon inspired optimization solving MKP}

After generating the encoding and decoding process for applying pigeon inspired optimization to the multidimensional knapsack problem, the all step for applying the PIO in MKP that we proposed is explained in pseudocode shown in Figure 2.

\section{RESULTS AND DISCUSSIONS}

\subsection{Numerical experiment instances}

The MKP cases that will be solved using PIO are benchmark instances that are often used in other studies. The MKP instances have also been implemented using Particle Swarm Algorithm (PSO) (Hembecker et al., 2007), Intelligent Water Drops Algorithm (IWD) (ShahHosseini, 2009), and Genetic Algorithm (GA) (Khuri et al., 1994), and the result of these three of researches will be compared with the result from this research using PIO.

There are 10 instances to be implemented in this chapter. In these 10 instances, 2 instances were taken from Senyu \& Toyada (1967) (it will be called as SENTO instances), and 8 instances were taken from Weingartner \& Ness (1967) (it will be called as WEING instances). Table 5 shows the problem characteristics for all instances to be implemented. 


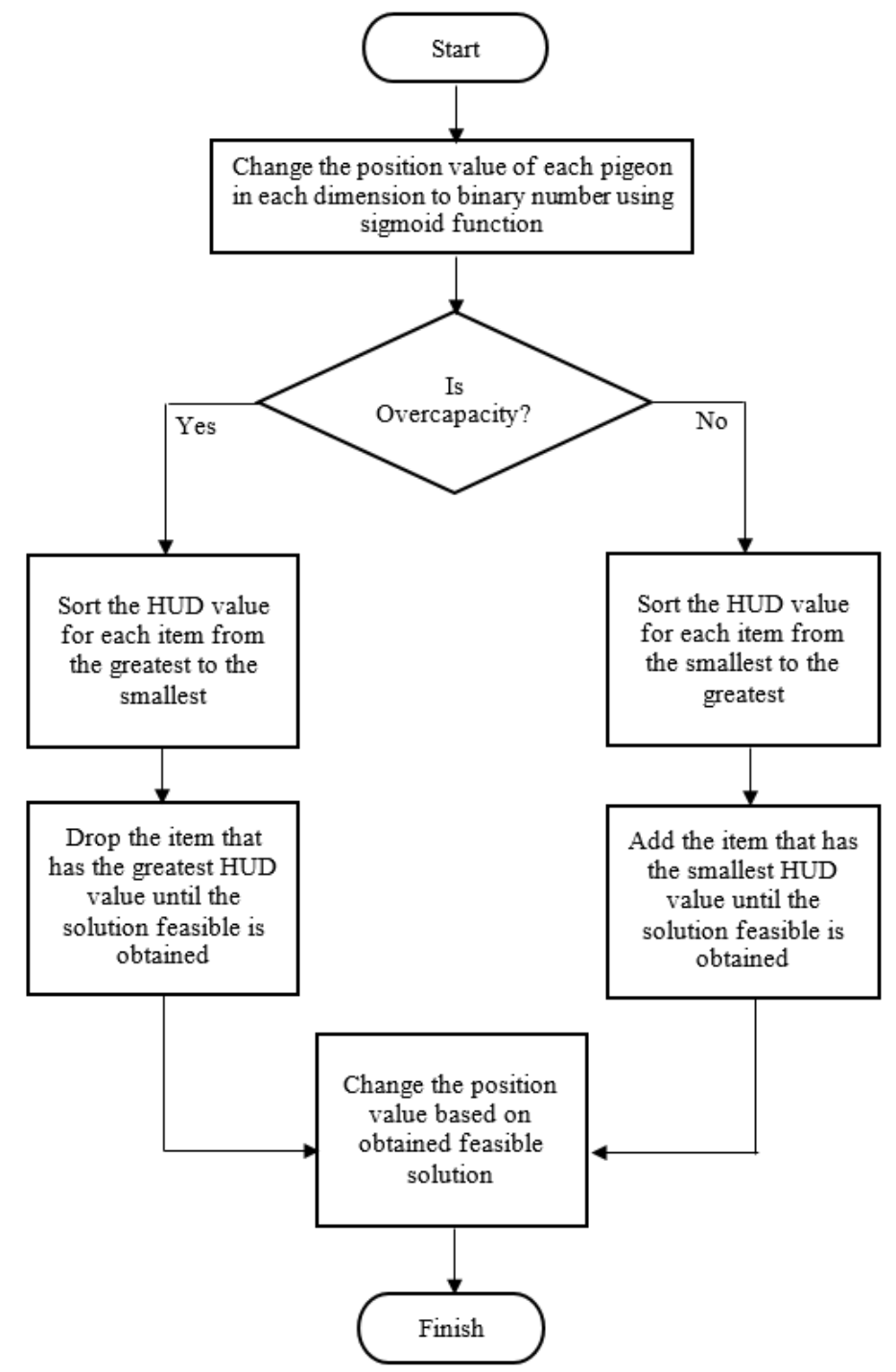

Figure 1. Drop/add items procedure

1. Input the data of MKP problem: the number of knapsacks, the number of items, the weight of each item, capacity of each knapsack, benefits of each item.

2. Input the value of parameters that are used in PIO: the number of iterations for map and compass operator ( $\mathrm{Nc} 1 \mathrm{max})$, the number of iterations for landmark operator ( $\mathrm{Nc} 2 \mathrm{max})$, the number of pigeons (Np), the number of dimensions (D), the value of map and compass operator factor $(\mathrm{R})$.

3. Calculate $\mathrm{HUD}_{\mathrm{i}}$ using equation (12).

4. Initialize $\mathrm{Vpd} 0$ by generating the random number between 0 and 1 .

5. Initialize Xpd0 by generating the random number between 0 and 1 .

6. Do drop/add item procedure.

7. Calculate the fitness value, fitnessB, fitnessg, and update $\mathrm{XB}$ and $\mathrm{Xg}$.

8. Do map and compass operator calculation.

9. If the number of iterations has not yet reached $\mathrm{Nc} 1 \mathrm{max}$ or other predetermined condition, back to step 8; otherwise, go to step 10 .

10. Do landmark operator calculation.

11. If the number of iterations has not yet reached $\mathrm{Nc} 2 \mathrm{max}$ or other predetermined condition, back to step 10, otherwise is finished. 


\subsection{Parameter setting of PIO}

Before implementing PIO on the instances, we determine the best parameter value of PIO to be used in this research. The determination of the PIO parameter will be done using one factor at a time (OFAT). The initial value used in this research is taken from the parameter value from the initial paper on PIO by Duan and Qiao in 2014). The initial value of these parameters are $\mathrm{Nc} 1 \max =150, \mathrm{Nc} 2 \max =8, \mathrm{~Np}=150$ and $\mathrm{R}=0.2$. The application of OFAT in this paper is done by five replications. There are only two cases that will be experimented with to get the best parameter, namely SENTO1 and WEING7 cases, because those two cases are the most complex than others. The first parameter to be tested is NC1max. In the experiment, the value of Nc1max will be subtracted and added by a multiple of 100. In running the program, there are 21 levels or treatments for changing the value of the Nc1max parameter. Table 6 and 7 below shows the combination of parameters for changing the value of the Nc1max parameter in the SENTO1 case and the result from this parameter combination.

Based on table 11 above, the Nc1max parameter of 1450 yields the highest performance, so the best value of the Nc1max parameter is 1450 for SENTO 1 case. This NC1max best value then will be used on the determination of the remaining other parameters. After the best value of the Nc1max parameter is obtained, then the best parameter value for the other PIO parameter will be determined using the same fashion. Np parameter value will also be subtracted and added by a multiple of 100. Nc2max values in this experiment are in the range of 1 to 9 . $\mathrm{R}$ parameter values in this experiment are in the range of 0.1 to 1 , with an increase of 0.1 in every treatment.

Based on these experiments, we get that the best parameter combination value of the PIO parameter for SENTO1 case are Nc1 $\max =1450, \mathrm{Nc} 2 \max =6, \mathrm{~Np}=$ 450 and $\mathrm{R}=0.3$. After the best parameter combination value of the PIO parameter for SENTO 1 has been determined, we determine the best parameter combination value of the PIO parameter for WEING 7 in the same way that we have done in SENTO 1. The best parameter combination value of the PIO parameter for WEING7 case are $\mathrm{Nc} 1 \max =1150, \mathrm{Nc} 2 \mathrm{max}=4, \mathrm{~Np}$ $=300$ and $\mathrm{R}=0.8$.

\subsection{Numerical experiment}

Based on the parameter setting above, it is obtained two combinations of a parameter that yield the best performance of PIO. The first combination is Nc1 max $=$ 1450, $\mathrm{Nc} 2 \max =6, \mathrm{~Np}=450$ and $\mathrm{R}=0.3$ and the second combination is $\mathrm{Nc} 1 \max =1150, \mathrm{Nc} 2 \max =4, \mathrm{~Np}=300$ and $\mathrm{R}=0.8$. Both combinations will be applied to all 10 cases in this research. The result of PIO implementation for all ten instances using the first parameter combination is shown in Table 8 below.

Table 9 below shows the result of PIO implementation for all ten instances using the second parameter combination.
After both parameters, combinations are used in PIO implementation for all ten instances, and the result is obtained, we will choose the best value from one of two parameter combinations. Table 10 below shows the summary of the implementation of two-parameter combinations and the best value from one of the twoparameter combinations.

Performance comparison is made by comparing the best objective function on all ten instances with the result obtained by other metaheuristics. As stated before, the other metaheuristic that will be compared with PIO are particle swarm optimization (PSO), intelligent water drops (IWD), and genetic algorithm (GA). Table 11 and 12 below shows the performance comparison of PIO, PSO, IWD, and GA in all ten instances.

As we see in Table 11, the best known is the best value that has ever known to date. IWD is not applied to SENTO1 and SENTO2 instances, while GA is not applied to WEING1, WEING2, WEING3, WEING4, WEING5, and WEING6 instances. PSO is applied to all ten instances. From the result above, it can be shown that the performance of PIO is a satisfactory result compared to all the compared metaheuristics.

\section{CONCLUSIONS}

Pigeon-inspired optimization (PIO) can be applied to Multidimensional Knapsack Problem (MKP) using a random number coding method for initialization and binary number from sigmoid functions for the decoding process. In this encoding and decoding process, HUD is used, which is one of the simplest local heuristic methods to manage the constraints of the MKP. From the coding method and the decode is then translated into the position of the pigeon on the map and the compass operator and the landmark operator, from which the position value will be calculated the objective function value of fitness as well as the global position of the global pigeon which determines which items will be chosen into a knapsack.

In the implementation of the PIO algorithm to the ten instances, it is known that overall the performance of the PIO algorithm is better than the performance of the PSO algorithm and the performance of the GA algorithm in its application to MKP. However, this PIO algorithm gets slightly worse results than the IWD algorithm in its application to MKP. For further research, it is possible to apply the PIO algorithm to solve other discrete problems and combinatorial problems. 
Table 6: Parameter combination by changing NC1max parameter on SENTO1 case

\begin{tabular}{|c|c|c|c|c|}
\hline Parameter combination & Nc1max & Nc2max & Np & $\mathrm{R}$ \\
\hline 1 & 50 & 8 & 150 & 0,2 \\
\hline 2 & 150 & 8 & 150 & 0,2 \\
\hline 3 & 250 & 8 & 150 & 0,2 \\
\hline 4 & 350 & 8 & 150 & 0,2 \\
\hline 5 & 450 & 8 & 150 & 0,2 \\
\hline 6 & 550 & 8 & 150 & 0,2 \\
\hline 7 & 650 & 8 & 150 & 0,2 \\
\hline 8 & 750 & 8 & 150 & 0,2 \\
\hline 9 & 850 & 8 & 150 & 0,2 \\
\hline 10 & 950 & 8 & 150 & 0,2 \\
\hline 11 & 1050 & 8 & 150 & 0,2 \\
\hline 12 & 1150 & 8 & 150 & 0,2 \\
\hline 13 & 1250 & 8 & 150 & 0,2 \\
\hline 14 & 1350 & 8 & 150 & 0,2 \\
\hline 15 & 1450 & 8 & 150 & 0,2 \\
\hline 16 & 1550 & 8 & 150 & 0,2 \\
\hline 17 & 1650 & 8 & 150 & 0,2 \\
\hline 18 & 1750 & 8 & 150 & 0,2 \\
\hline 19 & 1850 & 8 & 150 & 0,2 \\
\hline 20 & 1950 & 8 & 150 & 0,2 \\
\hline 21 & 2050 & 8 & 150 & 0,2 \\
\hline
\end{tabular}

Table 7: The result of parameter combination by changing NC1max parameter on SENTO1 case

\begin{tabular}{|c|c|c|c|c|c|c|c|c|}
\hline \multirow{2}{*}{$\begin{array}{l}\text { Parameter } \\
\text { combination }\end{array}$} & \multicolumn{5}{|c|}{ Replication } & \multirow[t]{2}{*}{ Best } & \multirow[t]{2}{*}{ Average } & \multirow[t]{2}{*}{ STDEV } \\
\hline & 1 & 2 & 3 & 4 & 5 & & & \\
\hline 1 & 7566 & 7586 & 7598 & 7586 & 7588 & 7598 & 7584.8 & 11.63 \\
\hline 2 & 7617 & 7622 & 7642 & 7641 & 7576 & 7642 & 7619.6 & 26.80 \\
\hline 3 & 7623 & 7643 & 7598 & 7598 & 7598 & 7643 & 7612 & 20.43 \\
\hline 4 & 7654 & 7622 & 7632 & 7643 & 7637 & 7654 & 7637.6 & 11.97 \\
\hline 5 & 7598 & 7639 & 7598 & 7622 & 7625 & 7639 & 7616.4 & 17.98 \\
\hline 6 & 7654 & 7598 & 7598 & 7615 & 7599 & 7654 & 7612.8 & 24.14 \\
\hline 7 & 7654 & 7641 & 7598 & 7635 & 7643 & 7654 & 7634.2 & 21.37 \\
\hline 8 & 7598 & 7654 & 7636 & 7632 & 7642 & 7654 & 7632.4 & 20.95 \\
\hline 9 & 7664 & 7599 & 7598 & 7674 & 7625 & 7674 & 7632 & 35.64 \\
\hline 10 & 7624 & 7637 & 7626 & 7625 & 7625 & 7637 & 7627.4 & 5.41 \\
\hline 11 & 7637 & 7660 & 7642 & 7651 & 7654 & 7660 & 7648.8 & 9.26 \\
\hline 12 & 7636 & 7632 & 7598 & 7635 & 7692 & 7692 & 7638.6 & 33.78 \\
\hline 13 & 7598 & 7598 & 7625 & 7679 & 7679 & 7679 & 7635.8 & 40.95 \\
\hline 14 & 7636 & 7626 & 7606 & 7679 & 7692 & 7692 & 7647.8 & 36.36 \\
\hline 15 & 7642 & 7642 & 7636 & 7625 & 7719 & 7719 & 7652.8 & 37.65 \\
\hline 16 & 7637 & 7622 & 7679 & 7636 & 7654 & 7679 & 7645.6 & 21.85 \\
\hline 17 & 7632 & 7637 & 7664 & 7632 & 7635 & 7664 & 7640 & 13.58 \\
\hline 18 & 7654 & 7637 & 7636 & 7634 & 7598 & 7654 & 7631.8 & 20.52 \\
\hline 19 & 7643 & 7599 & 7625 & 7637 & 7635 & 7643 & 7627.8 & 17.36 \\
\hline 20 & 7598 & 7651 & 7684 & 7642 & 7609 & 7684 & 7636.8 & 34.40 \\
\hline 21 & 7692 & 7632 & 7692 & 7598 & 7654 & 7692 & 7653.6 & 40.33 \\
\hline
\end{tabular}


Table 8: PIO Implementation using first parameter combination

\begin{tabular}{|c|c|c|c|c|c|c|}
\hline \multirow{2}{*}{ Instances } & \multicolumn{7}{|c|}{ Replication } & \\
\cline { 2 - 7 } & 1 & 2 & 3 & 4 & 5 & Best \\
\hline SENTO1 & 7679 & 7679 & 7719 & 7679 & 7679 & 7719 \\
\hline SENTO2 & 8619 & 8653 & 8645 & 8637 & 8613 & 8653 \\
\hline WEING1 & 141148 & 141278 & 141148 & 141148 & 141258 & 141278 \\
\hline WEING2 & 130103 & 130723 & 130723 & 130123 & 130163 & 130723 \\
\hline WEING3 & 95677 & 95007 & 95007 & 95467 & 95137 & 95677 \\
\hline WEING4 & 119337 & 119337 & 119337 & 119337 & 119337 & 119337 \\
\hline WEING5 & 98396 & 98396 & 98495 & 98396 & 98396 & 98495 \\
\hline WEING6 & 130123 & 130113 & 130113 & 130113 & 130113 & 130123 \\
\hline WEING7 & 1094452 & 1094417 & 1094356 & 1094547 & 1095007 & 1095007 \\
\hline WEING8 & 612430 & 614706 & 611555 & 612737 & 615315 & 615315 \\
\hline
\end{tabular}

Table 9. PIO Implementation using second parameter combination

\begin{tabular}{|c|c|c|c|c|c|c|}
\hline \multirow{2}{*}{ Instances } & \multicolumn{7}{|c|}{ Replication } & \\
\cline { 2 - 7 } & 1 & 2 & 3 & 4 & 5 & Best \\
\hline SENTO1 & 7719 & 7679 & 7679 & 7679 & 7679 & 7719 \\
\hline SENTO2 & 8649 & 8619 & 8618 & 8599 & 8665 & 8665 \\
\hline WEING1 & 141148 & 141258 & 141148 & 141148 & 141148 & 141258 \\
\hline WEING2 & 130103 & 130712 & 130103 & 130883 & 130103 & 130883 \\
\hline WEING3 & 95007 & 95007 & 95007 & 95007 & 95007 & 95007 \\
\hline WEING4 & 119337 & 119337 & 119337 & 119337 & 119337 & 119337 \\
\hline WEING5 & 98495 & 98396 & 98396 & 98396 & 98396 & 98495 \\
\hline WEING6 & 130623 & 130202 & 130123 & 130113 & 130123 & 130623 \\
\hline WEING7 & 1094262 & 1094811 & 1094467 & 1094111 & 1094356 & 1094811 \\
\hline WEING8 & 612880 & 611100 & 617901 & 614553 & 613751 & 617901 \\
\hline
\end{tabular}

Table 10: PIO Implementation using both parameter combination

\begin{tabular}{|c|c|c|c|}
\hline \multirow{2}{*}{ Instances } & \multicolumn{2}{|c|}{ Best value of two-parameter combinations } & \multirow{2}{*}{ Best } \\
\cline { 2 - 3 } & 1 & 2 & \\
\hline SENTO1 & 7719 & 7719 & 7719 \\
\hline SENTO2 & 8653 & 8665 & 8665 \\
\hline WEING1 & 141278 & 141258 & 141278 \\
\hline WEING2 & 130723 & 130883 & 130883 \\
\hline WEING3 & 95677 & 95007 & 95677 \\
\hline WEING4 & 119337 & 119337 & 119337 \\
\hline WEING5 & 98495 & 98495 & 98495 \\
\hline WEING6 & 130123 & 130623 & 130623 \\
\hline WEING7 & 1095007 & 1094811 & 1095007 \\
\hline WEING8 & 615315 & 617901 & 617901 \\
\hline
\end{tabular}


Table 11: Performance comparison of PIO, PSO, IWD, GA in all ten instances

\begin{tabular}{|c|c|c|c|c|c|}
\hline Instances & PSO & IWD & GA & PIO & Best Known \\
\hline SENTO1 & 7,725 & - & 7,626 & 7,719 & 7,772 \\
\hline SENTO2 & 8,716 & - & 8,685 & 8,665 & 8,722 \\
\hline WEING1 & 138,927 & 141,278 & - & 141,278 & 141,278 \\
\hline WEING2 & 125,453 & 130,883 & - & 130,883 & 130,883 \\
\hline WEING3 & 92,297 & 95,677 & - & 95,677 & 95,677 \\
\hline WEING4 & 116,622 & 119,337 & - & 119,337 & 119,337 \\
\hline WEING5 & 93,678 & 98,796 & - & 98,495 & 98,796 \\
\hline WEING6 & 128,093 & 130,623 & - & 130,623 & 130,623 \\
\hline WEING7 & $1,059,560$ & $1,094,736$ & $1,093,987$ & $1,095,007$ & $1,095,445$ \\
\hline WEING8 & 492,347 & 620,872 & 613,383 & 617,901 & 624,319 \\
\hline
\end{tabular}

Table 12: Performance comparison of PIO, PSO, IWD, GA in percentage to best known

\begin{tabular}{|r|r|c|c|r|}
\hline Instances & PSO & IWD & GA & \multicolumn{1}{c|}{ PIO } \\
\hline SENTO1 & $99.40 \%$ & - & $98.12 \%$ & $99.32 \%$ \\
\hline SENTO2 & $99.93 \%$ & - & $99.58 \%$ & $99.35 \%$ \\
\hline WEING1 & $98.34 \%$ & $100.00 \%$ & - & $100.00 \%$ \\
\hline WEING2 & $95.85 \%$ & $100.00 \%$ & - & $100.00 \%$ \\
\hline WEING3 & $96.47 \%$ & $100.00 \%$ & - & $100.00 \%$ \\
\hline WEING4 & $97.72 \%$ & $100.00 \%$ & - & $100.00 \%$ \\
\hline WEING5 & $94.82 \%$ & $100.00 \%$ & - & $99.70 \%$ \\
\hline WEING6 & $98.06 \%$ & $100.00 \%$ & - & $100.00 \%$ \\
\hline WEING7 & $96.72 \%$ & $99.94 \%$ & $99.87 \%$ & $99.96 \%$ \\
\hline WEING8 & $78.86 \%$ & $99.45 \%$ & $98.25 \%$ & $98.97 \%$ \\
\hline
\end{tabular}

\section{REFERENCES}

Balev, S., Yanev, N., Freville, A., Andonov, R. (2008). A Dynamic Programming Based Reduction Procedure for the Multidimensional 0-1 Knapsack Problem. European Journal of Operational Research, 186 (1), 63-76.

Banati, H. \& Bajaj, M. (2011). Firefly Based Feature Selection Approach. International Journal of Computer Science Issues, 8(4), 473-480.

Basset, M.A., Doaa, E.-S., Faris, H., Mirjalili, S. (2019). A Binary Multi-Verse Optimizer for 0-1 Multidimensional Knapsack Problems with Application in Interactive Multimedia Systems. Computers \& Industrial Engineering, 132, 187-206.

Basset, M.A., El-Shahat, D., El-Henawy, I., Sangaiah, A.K. (2018). A Modified Flower Pollination Algorithm for the Multi-dimensional Knapsack Problem: Human-Centric Decision Making. Soft Computing, 22, 4221-4239.

Battiti, R. \& Tecchiolli, G. (1994). The reactive tabu search. ORSA Journal on Computing, 6, 126-140.
Berberler, M.E., Guler, A., Nuriyev, U.G. (2013). A Genetic Algorithm to Solve the Multi-dimensional Knapsack Problem. Mathematical and Computational Applications, 18(3), 486-494.

Bertsimas, D. \& Demir, R. (2002). An Approximate Dynamic Programming Approach to Multidimensional Knapsack Problem. Management Science, 48, 550-565.

Bolaji, A.L., Babatunde, B.S., Shola, P.B. (2017). Adaptation of Binary Pigeon-Inspired Algorithm for Solving Multidimensional Knapsack Problem. Soft Computing: Theories and Applications, 743-751.

Bolaji, A.L., Okwonu, F.Z., Shola, P.B., Balogun, B.S., Adubisi, O.D. (2020). A Modified Binary PigeonInspired Algorithm for Solving the Multi-dimensional Knapsack Problem. Journal of Intelligent Systems, 30(1), 90-103.

Chen, W.-N., Zhang, J., Chung, H.S.H., Zhong, W.-L., Wu, W.-G., Shi, Y.-H. (2010). A Novel Set-Based Particle Swarm Optimization Method for Discrete Optimization Problems. IEEE Transactions on Evolutionary Computation, 14(2), 278-300. 
Chih, M. (2015). Self-Adaptive Check and Repair Operator-Based Particle Swarm Optimization for the Multidimensional Knapsack Problem. Applied Soft Computing, 26, 378-389.

Chu, P.C. \& Beasley, J.E. (1998). A Genetic Algorithm for the Multidimensional Knapsack Problem. Journal of Heuristics, 4, 63-86.

Dammeyer, F. \& Voß, S. (1993). Dynamic Tabu List Management Using the Reverse Elimination Method. Annals of Operations Research, 41(2), 29-46.

Deng, Y. \& Duan H. (2016). Control Parameter Design for Automatic Carrier Landing System via PigeonInspired Optimization. Nonlinear Dynamics, 85, $97-$ 106.

Dou, R. \& Duan, H. (2016). Pigeon Inspired Optimization Approach to Model Prediction Control for Unmanned Air Vehicles. Aircraft Engineering and Aerospace Technology: An International Journal, 88 (1), 108-116.

Duan, H. \& Li, C. (2014). Target Detection Approach for UAVs via Improved Pigeon-Inspired Optimization and Edge Potential Function. Aerospace Science and Technology, 39, 352-360.

Duan, H. \& Qiao, P. (2014). Pigeon Inspired Optimization: A New Swarm Intelligence Optimizer for Air Robot Planning. International Journal of Intelligent Computing and Cybernetics, 7, 24-37.

Fingler, H., Caceres, E.N., Mongelli, H., Song, S.W. (2014). A Cuda Based Solution to the Multidimensional Knapsack Problem Using the Ant Colony Optimization. Procedia Computer Science, 29, 84-94.

Freville, A. \& Plateau, G. (1986). Heuristics and Reduction Methods for Multiple Constraints 0-1 Linear Programming Problems. European Journal of Operational Research, 24, 206-215.

Gilmore, P.C. \& Gomory, R. (1966). The Theory and Computation of Knapsack Functions. Operations Research, 14(6), 1045-1074.

Glover, F. \& Kochenberger, G.A. (1996). Critical Event Tabu Search for Multidimensional Knapsack Problem. Meta-heuristics, Springer, 407-202.

Haddar, B., Khemakhem, M., Hanafi, S., Wilbaut, C. (2016). A Hybrid Quantum Particle Swarm Optimization for the Multidimensional Knapsack Problem. Engineering Applications of Artificial Intelligence, 55, 1-13.

Hembecker, F., Lopes, H.S., \& Godoy Jr., W. (2007).
Particle Swarm Optimization for the Multidimensional Knapsack Problem. Proceedings of the Eighth International Conference on Adaptive and Natural Computing Algorithms, 4431, 358-365.

Ke, L., Feng, Z., Ren, Z., Wei, X. (2010). An Ant Colony Optimization Approach for the Multidimensional Knapsack Problem. Journal of Heuristics, 16(1), 6583.

Khuri, S., Back, T., \& Heitkotter, J. (1994). The Zero/One Multiple Knapsack Problem and Genetic Algorithms. Proceedings of the ACM Symposium on Applied Computing (SAC'94).

Kong, M., \& Tian, P. (2006). Apply the Particle Swarm Optimization to the Multi-dimensional Knapsack Problem, In: Rutkowski, L., Tadeusiewicz, R., Zadeh, L., Zurada, J. (Eds.), Artificial Intelligence and Soft Computing-ICAISC 2006, Vol 4029 of Lecture Notes in Computer Science, Springer Berlin Heidelberg, $1140-1149$.

Kong, M., Tian, P., Kao, Y. (2008). A New Ant Colony Optimization Algorithm for the Multidimensional Knapsack Problem. International Journal of Applied Metaheuristic Computing, 3(4), 43-63.

Ktari, R. \& Chabchoub, H. (2013). Essential Particle Swarm Optimization Queen with Tabu Search for MKP Resolution. Computing, 95(9), 897-921.

Lai, X., Hao, J.-K., Glover, F., Lu, Z. (2018). A Two Phase Tabu Evolutionary Algorithm for the 0-1 Multidimensional Knapsack Problem. Information Sciences, 436-437.

Leung, S.C., Zhang, D., Zhou, C., Wu, T. (2012). A Hybrid Simulated Annealing Meta-heuristic algorithm for the Two-Dimensional Knapsack Packing Problem. Computer and Operations Research, 39(1), 64-73.

Manzini, R., Speranza, M.G. (2012). Coral: An Exact Algorithm for the Multi-dimensional Knapsack Problem. INFORMS Journal of Computing, 24(3), 399-415.

Martello, S. \& Toth, P. (1990). Knapsack Problems: Algorithms and Computer Implementations. Chicester: John Wiley \& Sons Ltd.

Martins, J.P., Longo, H., Delbern, A.C. (2014). On the Effectiveness of Generic Algorithms for the Multidimensional Knapsack Problem. Proceedings of the 2014 conference companion on Genetic and evolutionary computation companion, ACM, 73-74.

Palit, S., Sinha, S.N., Molla, M.A., Khanra, A., Kule, M. (2011). A Cryptanalytic Attack on the Knapsack Cryptosystem using Binary Firefly Algorithm. Second International Conference on Computer and 
Communication Technology, 428-432.

Rezoug, A., Boughaci, D., Rezoug, A. (2015) Stochastic Local Search Combined with Simulated Annealing for the $0=1$ Multi-dimensional Knapsack Problem. Symposium on Complex Systems and Intelligent Computing (CompSIC).

Senyu, S. \& Toyada, Y. (1967). An Approach to Linear Programming With 0-1 Variables. Management Science, 15(4), 196-207.

Shah-Hosseini, H. (2009). The Intelligent Water Drops Algorithm: A Nature-inspired Swarm-based Optimization Algorithm. International Journal of BioInspired Computation, 1, 71-79.

Shih, W. (1979). A Branch and Bound Method for the Multiconstraint Zero-one Knapsack Problem. Journal of the Operational Research Society, 15, 196-207.

Tisna, A., F.D., Abusini, S., Andar, A. (2013). Hybrid Greedy-Particle Swarm Optimization-Genetic Algorithm and Its Convergence to Solve Multidimensional Knapsack Problem 0-1. Journal of Theoritical and Applied Information Technology, 58(3), 522-528.

Vasquez, M. \& Hao, J.-K. (2001). Une Approche hybride pur le sac a dos multi-dimensionnel en variales 0-1. Operations Research, 35(4), 415-438.

Vasquez, M. \& Vimont, Y. (2005) Improved Results on the 0-1 Multi-dimensional Knapsack Problem. European Journal of Operational Research, 165(1), 70-81.

Vimont, Y., Boussier, S., Vasquez, M. (2008). Reduced Cost Propagation in an Efficient Impicit Enumeration for the 0-1 Multi-dimensional Knapsack Problem. Journal of Combinatorial Optimization, 15(2), 165 178.

Wan, N.F. \& Nolle, L. (2009). Solving a Multidimensional Knapsack Problem Using a Hybrid Particle Swarm Optimization Algorithm. Proceedings of the 23rd European Conference on Modelling and Simulation, ECMS 2009.

Weingartner, H.M., \& Ness, D.N. (1967). Methods for the Solution of the Multi-dimensional 0/1 Knapsack Problem. Operation Research, 15(1), 83-103.

Zhang, X., Wu, C., Li, J., Wang, X., Yang, Z., Lee, J.M., Jung K.-H. (2016). Binary Artificial Algae Algorithm for Multidimensional Knapsack Problem. Applied Soft Computing, 43, 583-595. 
This page is intentionally left blank 